\title{
The Architecture of an Internet-based Virtual Industrial Community
}

\author{
Mingwei Zhou \\ CSIRO Manufacturing Science and Technology, Australia \\ Email: Mingwei.Zhou@cmst.csiro.au
}

Key words Enterprise Integration, Enterprise Engineering, Virtual Industrial Community, Virtual Manufacturing Enterprise, Information Infrastructure

\begin{abstract}
Virtual Enterprise (VE) has been increasingly seen as a promising solution for global manufacturing. This paper discusses the environment for supporting the lifecycle of virtual enterprises, from their creation, operation, to decommission. The concept of an internet based "Virtual Industrial Community" has been proposed to depict such kind of environment where potential member companies of virtual enterprises are facilitated with a virtual "community" or "industrial park" like infrastructure. The aims of VIC are to enable these companies to organise or participate in a virtual enterprise more conveniently and efficiently, and to stabilise the dynamics in the design and operation of a virtual enterprise.
\end{abstract}

\section{INTRODUCTION}

With the globalisation of economies, manufacturing enterprises are facing more and more rapid changes in marketplace, manufacturing practice, organisational structure, and information infrastructure. To seize and maintain its competitive advantage, a manufacturing enterprise must be able to quickly react to changes in their business. As a consequence, a new business paradigm of Virtual Enterprise (VE) has been evolved as a promising solution for global manufacturing. A Virtual Manufacturing Enterprise (VME) is usually referred to as a temporary consortium of independent companies which come together to quickly exploit fastchanging global manufacturing opportunities.

VME has been an active research topic in the last few years. A significant understanding on the architectures of VME has been achieved $[1,2,6,11]$. The tools and methods necessary for the design and operation of 
VME have been proposed [3,4,7,10], and various software platforms for supporting the operation and management of VME have also been implemented [7, 12].

Current research has been mainly focused on the supporting of the operation of a VME, that is, the operation of a virtual enterprise in the real business environment. However, not much has been done on the supporting the operation of a real enterprise in a virtual business environment, which is the situation when a potential company is looking for opportunities to form or join a virtual enterprise.

We have found that the later (the operation of a real enterprise in a virtual business environment) in fact needs urgent attention, as it is the prerequisites for the former (the operation of a virtual enterprise in the real business environment). We identified that many obstacles for the effective formation and operation of a VME, such as the lack of confidence and trust, the lack of the identity and ownership, and the fear of losing uniqueness and know-how [5,13], are mainly due to the lack of a clear overall view of the virtual business environment.

The work on Virtual Industrial Community (VIC) is to investigate on the potential architectures of such a virtual business environment where the real companies can create and join a virtual enterprise, as well as the necessary information infrastructures for supporting the VIC.

\section{VIRTUAL INDUSTRIAL COMMUNITY}

The IMS Globeman'21 project [14] identified a common VE framework based on the GERAM (Generic Enterprise Reference Architecture and Methodology) [3]. The framework consists of three recursive lifecycles: network, VE, and product. These lifecycles indicate that the operation of the enterprise network leads to the creation, operation, and decommission of a $\mathrm{VE}$, and the operation of the VE leads to the creation, operation, and implementation of the product. As show in Figure 1.

Here, the enterprise network is a very flexibly defined entity: it could be in the form of a simple directory service (such as a mailing list or a catalogue), or in the form of a fully incorporated temporary consortium. In most time, a network is only served as a candidate pool for a virtual enterprise. The network is usually non-exclusive, and a company may join multiple networks, therefore may end up with participation in competing virtual enterprises. There is very weak legal and financial binding, if there is any, within a network than that within a virtual enterprise. This often makes the operation of a network very dynamic, and sometimes, unstable. 
The aim of a Virtual Industrial Community (VIC) is to establish a more stable and long lasting virtual business environment that can accommodate multiple networks and virtual enterprises, and provide the whole lifecycle support to the virtual enterprises.

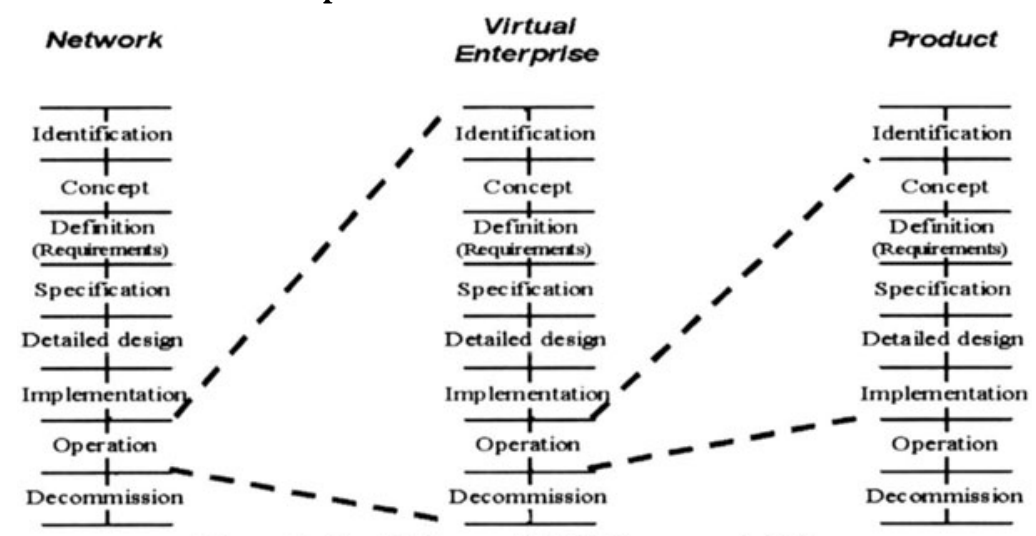

Figure 1: The Globeman'2I VE Framework [14]

A VIC, like a geographical community, is an open environment where any company may find a suitable place, and make connection with its neighbours by promoting and establishing its identity, and initiating a new network or joining any existing networks.

In addition to engineering and manufacturing companies, a VIC may have other companies providing various services such as IT, finance, accounting, legal, project management, and consulting. A VIC can be seen as an open network, or a network of networks, but in our opinion, a VIC should operate more like a normal community than a network. It provides services that a real industrial community such as the business centres and industrial parks.

\section{ARCHITECTURE}

A VIC has three major elements: the residents, the facilities, and the protocols. The residents of a VIC communicate to each other through their provided community facilities according to appropriate protocols (Figure 2).

The residents of a VIC consist of two types of companies: the corecompetence providers and the service providers. Some residents may fall into both categories (e.g., a fastener maker plays the role as a corecompetence provider when it is making a special purpose bolt, and as a service provider when it is making the standardised bolts). The corecompetence providers are mainly the engineering and manufacturing 
companies who are usually the initiators and participants of a VME or a network. While the service providers may include a wide range of companies such as banks and ASPs (Application Service Providers) who are usually not a member of a VME or network, but they may play the role as the organiser, manager, or consultant of the VE or network in the VIC. As the names indicate, the service providers provide services to the corecompetence providers.

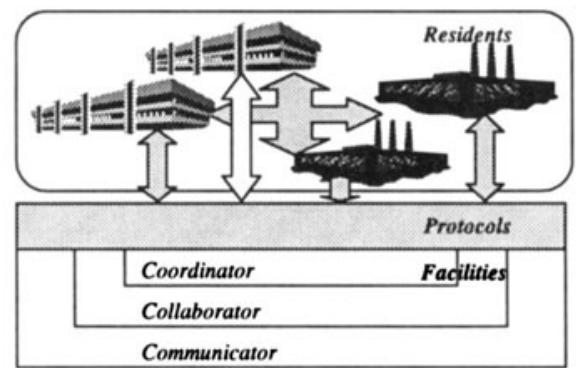

Figure 2: Architecture of a Virtual Industrial Community

The facilities of a VIC consist of three functional roles: the coordinator, the collaborator, and the communicator. These three roles were initially identified for supporting the lifecycle of a VME [12], and we find it is also applicable to VIC although their detailed functional specification may vary.

The VIC is managed through coordination instead of commanding. The coordinator is used to help establish trust, resolve conflict and make the VIC operate more efficiently. The coordinator provides the means for managing the company's identities, capabilities, capacities, and performances. It may also provide facilities for remotely monitoring and supervising the work-inprogress, as well as cross enterprise boundary scheduling and planning.

The VIC encourages and facilitates the collaboration among its residents. The collaborator will provide functions for exchanging and sharing of product and business information, enable the geographically distributed residents to work together more effectively, and provide the repository and warehouse for applications, models, data, and documents, serving as the memory of the VIC. The communicator provides a meeting place for the VIC. It also provides facilities such as identity verification, post office, chat room, bulletin board, and video conferencing.

The protocols are the spirit of the VIC. The protocols are those standardised or mutually agreed business processes that guide the coordination, collaboration, and communication among the VIC residents. The VIC protocols are implemented and supported by the VIC facilities (the coordinator, the collaborator, and the communicator). 


\section{CONCLUSION}

The work presented in this paper contributes to the concept of a Virtual Industrial Community (VIC). The architecture of the VIC is discussed, and the proposed VIC is expected to provide a more stable environment for support the lifecycle of virtual enterprise. The findings reported here represent our first step towards the understanding of the social and organisational behaviour of a virtual manufacturing enterprise, which will enable us to develop a software platform for supporting the design and operation of a virtual manufacturing enterprise.

\section{REFERENCES}

[1] Afsarmanesh $\mathrm{H}$ et al, Towards an Architecture for Virtual Enterprises, Esprit Prodnet Project, 1997

[2] Burnes P, Nemes L, and Williams TJ, Architectures for Enterprise Integration, Chapman and Hall, 1996

[3] Burnes $\mathrm{P}$ and Nemes L, Modelling and Methodologies for Enterprise Integration, Chapman and Hall, 1996

[4] Camarinha-Matos, LM, Lima C, Configuration and Coordination issues in a Virtual Enterprise Environment, in The Globalization of Manufacturing in the Digital Communications Era of the $21^{\text {st }}$ Century: Innovation, Agility and the Virtual Enterprise, 1998

[5] Westkamper, E, Manufacturing in Networks - Competitive Advantages for Virtual Enterprises, in The Globalization of Manufacturing in the Digital Communications Era of the $21^{s t}$ Century: Innovation, Agility and the Virtual Enterprise, 1998

[6] Hedberg B, Olve NG, Inside the Virtual Organisation, Strategic Management Society, October 1997

[7] Mills, JJ, Brand, M, and Elmasri, AeroWEB: An Information Infrastructure for the Supply Chain, in Information Infrastructure Systems for Manufacturing II, edited by Miles JJ. and Kimura F, Kluwer Academic Publishers, 1998,

[8] Neill H, Sackett P, The Extended Manufacturing Enterprise Paradigm, in Management Decision, 32(8):42-49

[9] NIIIP Consortium, U.S. National Industrial Information Infrastructure Protocols, Report No. NTR95-01, 1995

[10] Williams TJ, The Purdue Enterprise Reference Architecture, Instrument Society of America, North Carolina, 1991

[11] Zhou M., Enterprise Modelling Methodologies and Tools, IMS Globeman21 report, 1996

[12] Zhou M, et al, A framework for design a virtual manufacturing enterprise and its implementation as a workbench, in Information Infrastructure System for Manufacturing, edited by Miles J.J. and Kimura F., Kluwer Academic Publishers, 1998

[13] Zhou M, Neff P, Virtual Manufacturing Enterprises Design Using Enterprise Integration and Risk Management Methodologies, in The Proceedings of the International Conferences on Manufacturing Engineering 2000, Sydney, 2000

[14] IMS Globeman'21 Consortium, The Common Concept Report, 1999 